\title{
Should We Accept Arguments from Skeptics to Ignore the Psi Data? A Comment on Reber and Alcock's "Searching for the Impossible"
}

\author{
George R. Williams \\ grwilliams@gmail.com \\ Submitted October 4, 2019; Accepted October 10, 2019; Published December 30, 2019
}

DOI: https://doi.org/10.31275/2019/1681

Copyright: Creative Commons CC-BY-NC

\begin{abstract}
Reber and Alcock have recently made a sharp attack on the entire psi literature, and in particular a recent overview by Cardeña of the meta-analyses across various categories of psi. They claim the data are inherently flawed because of their disconnect with our current understanding of the world. As a result, they ignore the data and identify key scientific principles that they argue clash with psi. In this Commentary, I argue that these key principles are difficult to apply in areas where our understanding remains poor, especially quantum mechanics and consciousness. I also explore how the psi data may fit within these two domains.
\end{abstract}

\section{Introduction}

Recently, the journal American Psychologist published a paper by Etzel Cardeña that summarized the meta-analyses on various modes of psi and provided as well historical and theoretical background (Cardeña, 2018). Cardeña's paper is most notable with its comprehensive approach. The presented meta-analyses give us perhaps the best bird's-eye view of psi research to date. The combined studies include various modes or categories of psi, as well as different experimental designs for each one. In the paper, Cardeña claims that the overall evidence "provides cumulative support for the reality of psi, which cannot be readily explained away by the quality of the studies, fraud, selective reporting, experimental or analytical incompetence, or other frequent criticisms" (Cardeña, 2018, p. 1). He also notes that the rigor of the psi experimental methodology has increased with time, often including analyses for possible publication bias as well as the quality of the studies. 
Possibly because psi remains very controversial, American Psychologist followed with another paper, by Arthur Reber and James Alcock, that sharply criticized Cardeña's paper, as well as the field as a whole (Reber \& Alcock, 2019a). However, it would probably be more accurate to say that they dismissed all of the psi research that has been performed and published to date. In their critique, Reber and Alcock ignore the data Cardeña presents and instead argue why real psi effects simply cannot exist. Although they do not explicitly acknowledge that they ignored the data in their paper for American Psychologist, they do make this clear in a companion essay:

\begin{abstract}
We did not examine the data for psi, to the consternation of the parapsychologist who was one of the reviewers. Our reason was simple: The data are irrelevant. We used a classic, rhetorical device, adynaton, a form of hyperbole so extreme it is, in effect, impossible. Ours was "pigs cannot fly"hence data that show they can are the result of flawed methodology, weak controls, inappropriate data analysis, or fraud. (Reber \& Alcock, 2019b, p. 8)
\end{abstract}

Let's at least give them this: It's not every day we encounter an argument that invokes scientific principles to make the case for ignoring the data. Of course, this strategy of argument might worry anyone familiar with the history of science (or just about any aspect of science). But Reber and Alcock are unworried. They hinge their argument on what they view as the conflict between the psi data and four key scientific principles: causal mechanism, time's arrow, thermodynamics, and the inverse square law. The authors then proceed to discuss each of these areas of physics and how the psi data clash with it.

In my paper, I will focus on Reber and Alcock's (2019a) attack on psi, rather than on Cardeña's summary overview. In the next section, I'll take a closer look at their claims on the ways that psi conflicts with established scientific principles. I'll follow that section with more discussion of quantum mechanics. After that, I'll take a critical look at some of the ways that Reber and Alcock characterize the psi research. Next, I'll examine their argument that psi cannot be real on the basis of David Hume's argument against miracles. I'll follow this with a section on the persistent mystery of consciousness. A brief conclusion is provided at the end.

\title{
Psi versus Four Scientific Principles
}

Reber and Alcock (2019a) list four crucial ways that psi conflicts with our scientific understanding: 1) lack of a causal mechanism, 2) time reversal and the flipping of cause and effect, 3) violation the laws of thermodynamics, and 4) violations of the inverse square law. One red flag right off the bat is that Reber and Alcock do not justify any of these criteria from the 
philosophy of science literature that concerns the demarcation question. Perhaps they think these four principles are obvious and that everyone should agree on them. But the fact that Reber and Alcock make no attempt to fit it within the relevant literature raises the possibility that their list is an ad hoc construction designed only to attack psi, and has no relevance for science in general. If so, their justification for ignoring the psi data would seem precarious. And suppose there are non-psi theories that clashed with one or more of their selected scientific principles? Would they be prepared to attack them as well? In any case, let's consider each of these principles in turn.

Lack of causal mechanism. With regard to this first principle, the authors boldly declare: "Science is mechanistic; reliable phenomena are viewed in the context of bridging principles that allow for the identification of causal links for observed effects" (Reber \& Alcock, 2019a, p. 2). But one might wonder about cases where the mechanisms or causal links are not yet uncovered. That is, perhaps today we observe something that we cannot account for with a mechanism, but perhaps later we will. Can't we develop something preliminary or speculative, that eventually gets us toward a more developed theory? Reber and Alcock apparently rule this out, at least for psi. Unless the mechanism can be specified, or analyzed in the context of mechanisms we currently understand, the data must be faulty.

But strangely, just a few words later, Reber and Alcock pivot and recount the example of Newton's law of gravity and its apparent action at a distance. This was considered suspect, they explain, until Einstein's richer theory of general relativity came on the scene. So, on one hand, they argue that the psi data cannot be true because no causal mechanism has been identified. But their argument proceeds to include a famous example of a useful theory that did not specify a mechanism for a very long time. They go on to list other examples of preliminary theories that went on to be better developed and accepted over time. But because no causal mechanisms have been identified with parapsychology (at least so far), the experimental findings must be wrong. So, with this inconsistency right out of the gate, Reber and Alcock make a bit of a stumble.

But is it the case that all scientific theories simply must identify a causal mechanism? Perhaps this is a reasonable characterization for classical physics, but this is not the case with quantum mechanics. In the standard interpretation of quantum mechanics, you have a wave function that is described by the Schrödinger equation, until a measurement is taken, and then the wave function "collapses" into the experimental observations. ${ }^{1}$ No one has as yet suggested a mechanism for this wave function collapse. There are in fact so many unresolved questions (such as what counts as 
a measurement) around this notion of collapse that finding a mechanism might be the least pressing issue among quantum physicists.

Of course, there have been efforts to move away from the standard or Copenhagen interpretation. There are, for example, objective collapse theories, but these don't identify mechanisms of collapse either. There is also the hidden variables approach, which is usually associated with David Bohm's guidance equation. And as a matter of fact, Bohm opposed the idea that quantum systems could be understood in mechanistic terms. Consider his (along with his colleague Basil Hiley) description of a quantum system:

The relationship between parts of a system ... implies a new quality of wholeness [italics in original] of the entire system going beyond anything that can be specified solely in terms of the actual spatial relationships of all the particles. This is indeed the feature which makes the quantum theory go beyond mechanism of any kind. (Bohm \& Hiley, 1993, p. 58)

Another interesting area obviously relevant to the psi data is the area of consciousness. Currently there is no known mechanism for generating consciousness. As I'll discuss later, some philosophers of mind consider the possibility that consciousness is fundamental in some sense. Thus, there are at least two interesting areas of inquiry that apparently do not lend themselves to mechanistic frameworks: quantum mechanics and consciousness. And it so happens that the psi data appear to fall into the domains of both of these.

Time reversal and the flipping of cause and effect. Next, Reber and Alcock argue that the mode of psi known as precognition requires a sort of time reversal that turns around the essential order of cause and effect. With regard to precognition, the authors are quite categorical. "Nowhere in the rest of science," they claim, "not even quantum mechanics, where a host of strange effects like quantum entanglement are accepted as real, is such a notion even considered" (Reber \& Alcock, 2019a, p. 3).

But this is quite wrong. Cardeña mentions two influential quantum physicists who have considered exactly that: David Bohm and Henry Stapp. The physicist Bohm has proposed that quantum systems might be governed by an underlying nonlocal field, characterized by potentialities. Further, he has suggested that precognition may involve an ability to perceive these potentialities (Bohm, 1996, pp.131-132). That is, instead of information traveling backward in time, Bohm suggests we are simply aware of current probabilities of future events. Also, Henry Stapp (2017) makes a similar conjecture. However, Stapp proposes that precognition might reflect an ability to slightly bias the Born probabilities so that events unfold according to (perhaps unconscious) mental intention (Stapp, 2017, p. 77). 
It is also the case that Roger Penrose, a rather famous physicist and mathematician whom Reber and Alcock cite in their paper, has collaborated with Stuart Hameroff on a framework where conscious experience is generated through quantum processes within the brain. In their proposed model, conscious experience emerges from a sort of quantum computing within the brain's microtubules. But especially important for our purposes here is that Penrose and Hameroff have suggested that their model may be consistent with temporal anomalies of the sort reported by Bem (Hameroff \& Penrose, 2014, p. 63).

Reber and Alcock simply dismiss Sheehan's (2015) exploration of the possibilities of retrocausality. However, as Sheehan (2015) notes, there is currently no consensus on the right interpretation of quantum mechanics, and some interpretations do seem consistent with some notion of retrocausality. He specifically discusses Cramer's (1986) framework inspired from earlier work between John Wheeler and Richard Feynman. Cramer's transactional interpretation obviates the necessity of measurement to "collapse the wave function." According to his theory, observable quantum results are the result of a "handshake" of two waves of differing temporal orientation, one moving forward in time and the other one backward. Kastner (2012) proposes that these waves actually exist as possibilities outside of physical spacetime.

The violation of the laws of thermodynamics. Next, Reber and Alcock claim that psi violates the laws of thermodynamics in two broad classes of experiments. In the case of precognition, they argue that this class of experiment presumes "a substanceless future has an impact on choices made by a material human in the present." They note that this would require creating matter or energy out of a world "lacking ontological status and having no existential reality" (Reber \& Alcock, 2019a, p. 4). To my knowledge, no psi advocate (or anyone else) has proposed anything like this. (Reber and Alcock provide no citations on this.) I don't see how precognition entails that kind of story. In any case, I've already touched on a number of ways theories in quantum mechanics might support precognition.

These authors also claim that psychokenisis conflicts with the laws of thermodynamics. But they don't explain how. Instead, they move on to argue that the small effects require meta-analysis, which apparently for them makes it inherently suspect. ${ }^{2}$ They next argue that if psychokinesis were indeed real, it should be exploitable in casinos. This is a fair point, but not a compelling one. Casinos have drastically different environments from the laboratories producing the experimental results. And it seems reasonable to me that psychokinesis is sensitive to changes in the environment. Also, the evidence on psychokinesis appears largely attributable to especially 
talented participants. Further, the reported effects sizes are so small, using such anomalous perturbation would likely require sitting for a relatively long time. But casinos are not hospitable environments for maintaining calm and concentration over relatively long periods.

Violations of inverse square laws. Reber and Alcock also argue that the invariance of psi effects with respect to distance is also disqualifying. Of course, it is the case that an inverse square relationship generally characterizes all physical forces. However, quantum entanglement is not subject to such a constraint, and this fact has intrigued psi researchers. Could this be an important clue on the nature of psi? The skeptics are quick to dismiss the possibility. In their words: "There is no claim of a transmission of energy between the separated particles, only that they are "entangled" (Reber \& Alcock, 2019a, p. 4).

It is true that quantum entanglement as most physicists understand it does not allow for a novel form of information or energy transmission. But energy or information transmission might not be required for some forms of psi. Telepathy or remote viewing, for example, perhaps only involves nonlocal correlations between processes in our unconsciousness and the environment. And as I've noted above, quantum physicists such as Bohm and Stapp have considered that precognition and presentiment might reflect the ability to sense probabilities of future events. This more probabilistic aspect of reality would likely have nonlocal features. Thus, possible interpretations currently on the table could support some forms of psi despite such violations of the inverse square relationships.

In addition, Bohm (1986) explores a framework that would support nonlocal features in anomalous perturbation. In Bohm's implicate order framework, mental intention might influence the underlying quantum field, characterized by potentialities and "active information," and could in turn influence the behavior of the particles governed by that field. All in all, the nonlocal behavior of quantum systems appears to keep the door open for phenomena that do not follow inverse square relationships.

As I've noted, by choosing to simply ignore the data, Reber and Alcock are choosing a route that runs counter to the lessons that the history of science has shown us. Blocking out the data using a constructed set of principles seems highly problematic, given that our understanding of the world is incomplete. There are indeed good reasons to think that their preferred list cannot completely characterize the behavior of all facets of our reality. Further, their additional tendency to make odd or unnecessary assumptions around a given psi category in order to generate a clash with their principles doesn't inspire confidence. 


\section{On Quantum Mechanics}

A chief problem that Reber and Alcock face is that the psi data arguably fall into areas that we continue to struggle to understand, such as quantum mechanics and consciousness. As they apply their list of principles in ways that conflict with psi, they appear to rely on a rather narrow reading of quantum mechanics. But the underlying ontology of quantum mechanics remains murky. As I noted above, some interpretations of quantum mechanics might be quite friendly to the psi data.

Also, the authors appear excessively willing to impose constraints on the theory that have little grounding in what we currently understand. Consider this line of argument (Reber \& Alcock, 2019a, p. 5):

Quantum mechanics is a theory about processes that occur at the microphysical level of individual particles based on mathematical models [italics original]. It can be understood only from the point of view of the mathematics; its coherence is in the formulae, not in everyday macroreality. Physicist Richard Feynman famously noted on more than one occasion, "It is safe to say that nobody understands quantum mechanics" (1965, p. 129), and mathematical physicist Roger Penrose, who argued that consciousness emerges in quantum mechanical processes in microtubules in neural tissue, put it succinctly: "Quantum mechanics makes absolutely no sense" (Penrose, 1989).

Notice the weak support the above quotes provide for their arguments. While it's true that our current understanding of quantum mechanics is based on the formalism of the Schrödinger equation, the quotes from Feynman and Penrose emphasize what we don't understand rather than what we do. Then Reber and Alcock follow with this:

What both are expressing is that the axiomatic structure of quantum mechanics is grounded in the formalisms of logic and their representations are independent of any particular macroreality. (Reber \& Alcock, 2019a, p. 5)

I can't find anything like that in the quotes from Feynman and Penrose. In any case, this statement is simply wrong. The wave function within the Schrödinger equation is highly context-dependent on all aspects of the quantum system, including the experimental apparatus. Any decision on how we design an experiment or what item of interest to observe has significant effects on the structure of the wave function. ${ }^{3}$

The problem Reber and Alcock have with trying to pin down quantum mechanics in ways that rule out experimental evidence they dislike can be illustrated in a related debate regarding the role of quantum mechanics 
in biology, explored in McFadden and Al-Khalili (2014). They describe how a group of physicists at MIT, experts in quantum mechanics, became incredulous at research conducted at Berkeley on quantum behavior being found in conjunction with photosynthesis in plants. They believed the results to be impossible and quite hilarious, because the relatively warm and noisy environments within plants should be hostile to quantum behavior. However, they sent a colleague to investigate and he determined that the reports were accurate. Quantum coherence was key for transferring captured photon energy through such a relatively warm but highly organized interior of the leaf. In their book, McFadden and Al-Khalili (2016) describe how quantum behavior is expanding in other areas relevant to biological processes.

So, to characterize all of this, we have two non-physicists shouting from the hilltops that some things are absolutely impossible, while at the same time some well-regarded quantum physicists are exploring the possibility of those very things. Their refusal to examine the data is uncomfortably similar to the priests who refused to look through Galileo's telescope. ${ }^{4}$ It's not really clear they understand what the physicists they cite are saying. And among their cited physicists, I find only one who explicitly argues that psi is inconsistent with known science and what we are likely to know: the cosmologist Sean Carroll. But Carroll is an odd choice for advising us on distinguishing science and pseudoscience.

The authors note that Carroll (2008) has "shown that no physical force could account for the results supposedly found in studies of psychokinesis." Not exactly. Carroll (2008) argued in an essay for online Discovery Magazine (and his blog) that our current understanding of physics is not consistent with the spoon bending form of psychokinesis. But while he didn't address the forms of psychokinesis presented by Cardeña, Carroll appears to be a card-carrying psi skeptic, and it's more than likely he would generalize his argument to apply toward other forms of psychokinesis. In response to Damien Broderick's challenge to look at the psi evidence, Carroll replied:

Direct investigations into parapsychology are not completely irrelevant; however, given the fact that the phenomena are incompatible with the laws of physics that have been tested to exquisite precision in an enormous variety of circumstances, I think their relevance is pretty darn minuscule. [If] the choice is between believing in sloppy research/confirmation bias, etc., and believing that quantum field theory is violated in some tangible macroscopic way that has never been noticed in any physical experiment, I will surely choose the former. It's not really a close call. ... I gave an argumentwhich nobody has refuted, or seemingly even tried to-that telekinesis is incompatible with what we know about how nature works. Given that, I'm not going to waste my time looking into the claims to the contrary. Life is too short to take every claim seriously. (Broderick \& Goertzel, 2014, p. 26) 
Essentially, Carroll's (2008) argument is that "in the modern framework of fundamental physics, not only do we know certain things, but we have a very precise understanding of the limits of our reliable knowledge." $\mathrm{He}$ proceeds with a summary of what we now know about particles and forces and on the extreme unlikelihood of a new force arising due to the copious experiments we've conducted. After arguing the impossibility that current known forces might be responsible for psychokinesis, Carroll reasons that it also is extremely unlikely that any new force might exist to account for psychokinesis.

However, in other work, Carroll recognizes that we remain fundamentally ignorant on a great deal that happens in the domain of quantum mechanics, as well as how our consciousness might interact with it. Consider a recent essay by Carroll published in The New York Times (Carroll, 2019). There, Carroll acknowledged: "Physicists don't understand their own theory any better than a typical smartphone user understands what's going on inside the device." He goes on to characterize the mystery in the following way:

When we're not looking, they [quantum objects] exist in "superpositions" of different possibilities, such as being at any one of various locations in space. But when we look they suddenly snap into just a single location, and that's where we see them. We can't predict exactly what that location will be; the best we can do is calculate the probability of different outcomes.

The whole thing is preposterous. Why are observations special? What counts as an "observation," anyway? When exactly does it happen? Does it need to be performed by a person? Is consciousness [italics original] somehow involved in the basic rules of reality? Together these questions are known as the "measurement problem" of quantum theory.

Carroll here appears to acknowledge not only that we remain ignorant at a deep level about quantum mechanics, but that our consciousness might be involved in a subtle way. This view is rather difficult to reconcile with his hostility to looking at the psi data.

And Carroll is a controversial choice to cite on the nature of pseudoscience for another reason: He is a strong and well-known advocate of Everett's many worlds theory. Briefly, Everett argued thata we should not go beyond the Schrödinger equation in explaining quantum behavior. And according to his view, each possible observation is instantiated. We should interpret quantum mechanics therefore as a continuous branching of our universe into countless other universes. ${ }^{5}$ However, because these are separate, they cannot be observed or tested. One might think that this presents a falsification problem. Many physicists do think this, but not Carroll. He has argued that falsification is likely an overrated virtue for theories of a certain class (Carroll, 2018). ${ }^{6}$ 
Carroll has also explored some very interesting possibilities within the Everett framework. In an interview, Carroll discussed joint work with Alan Guth, where different universes might co-exist with different arrows of time (Sokol, 2016). Carroll reveals in the interview that an important motivation was the question of why time's arrow points in one direction. "There's no such thing, at a very deep level, that causes [must] precede effects," says Carroll (apparently demoting one of Reber and Alcock's key principles). The work is highly speculative, yet nevertheless explores how different pocket universes manifest time moving forward or backward. ${ }^{7}$

My aim here is not to criticize the many worlds interpretation, although it is true that I'm not an advocate. I do believe there is value in putting radical ideas on the table, especially in areas that resist our efforts to explain. That said, my view is that the weight we assign to likely explanations should be proportional to the evidence provided, and in that respect many worlds falls short. But here I am mainly curious how Carroll, an advocate with a radical theory with no evidence and who brushes aside issues of falsifiability, somehow manages to play a role as an authority on pseudoscience and psi, the latter for which he has little knowledge.

But now I'd like to consider how some of Carroll's theories might fare with the scientific principles that Reber and Alcock used to dismiss the psi data. Would they insist that Carroll identify a mechanism through which the universe continues to split? (I am unaware of any mechanism being introduced.) How would their arrow of time criteria work in a theory that posits different universes with time moving in different directions? (I've already noted that Carroll doesn't seem to hold the "arrow of time" at the same level of esteem as Reber and Alcock.) Also, I would very much like to know: Given the rather extraordinary claims of the multiverse theory, would Reber and Alcock insist on extraordinary evidence?

\section{A Poor Characterization of the Psi Literature}

We've seen that Reber and Alccok will likely have difficulty applying their key scientific principles in other areas of science we currently understand poorly. With that in mind, let's return to their characterization of the psi literature. As I've noted, they simply dismiss it in its entirety.

Claims of evidence for psi are announced, only to later fall into disregard. Theories are enunciated and later abandoned. Methodologies are introduced, found wanting, discarded, and sometimes recycled. Each new procedure is introduced with claims of success, followed by failures to replicate, followed in turn by the publication of meta-analyses that are claimed to rescue the effect of interest. As excitement about each new procedure wanes, a resurgence of interest develops when another, apparently successful procedure is reported. (Reber \& Alcock, 2019a, p. 1) 
There isn't much justification for this sweeping claim apart from a controversial report from the National Research Council (Druckman \& Swets, 1988). ${ }^{8}$ We also can note that this report precedes all of the metaanalyses that Cardeña presents. Just a little further, these psi skeptics press their case and assert:

The single feature that marks this extended period of research involving literally thousands of published papers, hundreds of conferences and meetings, and dozens of review volumes ... is that nothing has been learned.

By their telling, the whole enterprise has been a waste of time. But no citation is offered to substantiate the claim.

But later in the paper, they note that Greenhouse (1991), a statistician and psi critic, acknowledges that parapsychologists should not be held to a higher standard than other scientists. However, Reber and Alcock strongly disagree. They "dispute this proposition in the strongest of terms. When confronted with 'miraculous' claims, standard procedure is precisely the opposite" (Reber \& Alcock, 2019a, p. 6). But then what do we make of their claims that the psi results are ephemeral and unrepeatable? If their characterizations of psi being riddled by failures to replicate are accurate, why is it necessary to abandon normal standards of evaluation? Science depends crucially on a "let the chips fall where they may" attitude toward the evidence. Apparently, Reber and Alcock are not prepared to accept such terms here. But then how can science progress if we are allowed to shift the standards for evaluation whenever it suits us? Naturally, such a position raises troubling implications for scientific inquiry much broader than psi. Can such a position be defended by those seeking some authoritative role in scientific discourse?

This sort of rhetorical back-flip illustrates the nature of how these authors inaccurately characterize the literature. I'll focus on another troubling example regarding their treatment of Daryl Bem.

We should note that Bem's (2011) precognition experiments were unusually innovative in a number of respects. Bem specified nine different experiments that time reversed various well-known psychological functions. In one example, participants were asked to choose between two curtains which one would reveal an erotic picture. Unknown to the participants, the picture was selected randomly by the computer after participants made their selection. In another case, Bem reversed a psychological priming effect in order to investigate the effect of a subminal message on participantsbut after a selection was made by each participant (p. 633). Bem found statistical significance with eight of the nine time-reversed experiments. However, Bem also took the unusual step of making all of his software publicly available so that researchers could very quickly and easily get to 
work on replication. Eventually, 90 different studies were performed on Bem's precognition experiments. While Bem's findings were not replicated in every case, the overall meta-analysis showed small but very statistically significant effects. ${ }^{9}$

The meta-analysis also revealed an intriguing pattern: Experiments associated with what Kahneman terms "thinking fast" modes of cognition were statistically significant, while those associated with "thinking slow" weren't. "Thinking fast" modes of cognition are generally unconscious processes, while more conscious mediated modes are associated with "thinking slow." This suggests possible links with recently developed theories, such as first-sight and psi-mediated instrumental response, that integrate psi with unconscious cognitive processes.

Reber and Alcock paint a different story. They acknowledge that Bem's (2011) findings generated a great deal of attention, which they attribute in large part to his relatively strong stature from previous work. But they cast some doubt on his results by noting that replications were hard to come by. But this is immediately followed by acknowledging that the meta-analysis demonstrated real (albeit small) effects confirming Bem. They don't bother discussing the apparent contradiction. ${ }^{10}$

Rather than Bem's original study or the meta-analysis that followed, Reber and Alcock focus their attention on a short extract of an interview with Bem taken from Engber (2017), an article on Bem's research as well as its greater impact for psychology in general. I found Engber mildly critical of Bem's findings, but in a relatively even-handed and honest way. Reber and Alcock remove this extract from a short interview with Bem:

I'm all for rigor, but I prefer other people do it. I see its importance-it's fun for some people-but I don't have the patience for it. If you looked at all my past experiments, they were always rhetorical devices. I gathered data to show how my point would be made. I used data as a point of persuasion, and I never really worried about, "Will this replicate or will this not?"

But Reber and Alcock edited out a short sentence from the extract, as well as failed to provide some necessary context.

"I'm all for rigor," he continued, "but I prefer other people do it. I see its importance-it's fun for some people-but I don't have the patience for it." It's been hard for him, he said, to move into a field where the data count for so much. "If you looked at all my past experiments, they were always rhetorical devices. I gathered data to show how my point would be made. I used data as a point of persuasion, and I never really worried about, 'Will this replicate or will this not?'"

As you can see, the original includes a sentence clarifying that Bem was adjusting to the difficulties of changing into a field where more attention is 
paid to the underlying methods and data. His "past experiments," where his focus was not so much on replication, were those of his previous mainstream work. This point is reinforced in the original text as Engber adds immediately following the extract, "When Bem started investigating ESP, he realized the details of his research methods would be scrutinized with far more care than they had before." This is also an important recognition that psi studies typically receive more scrutiny than more conventional ones. And a mere three paragraphs down, Engber offered an illuminating exchange from a colleague of Bem:

"Credit to Daryl Bem himself," Leif Nelson told me. "He's such a smart, interesting man.... In that paper, he [Bem] actively encouraged replication in a way that no one ever does. He [Bem] said, 'This is an extraordinary claim, so we need to be open with our procedures.'. . It was a prompt for skepticism and action."

But Reber and Alcock leave out this admission that Bem acted aggressively to encourage replication. Thus, Reber and Alcock attempt to discredit Bem and the meta-analysis confirming his findings, not by finding flaws in the method or data, but through extracting an excerpt from an interview in such a way that suggests the opposite meaning from what was intended in the original text. We might ask, if psi is genuinely as bogus as these critics claim, why are such mischaracterizations necessary?

\section{Is Psi a Miracle?}

Let's recall Reber and Alcock's core argument against using conventional standards to test for psi: Psi should be seen as something miraculous. But what exactly is the basis for this claim? It is difficult to say, beyond their argument that the psi data are inconsistent with more conventional theories and frameworks. As I've noted, quantum mechanics and consciousness also deviate in fundamental ways from established frameworks, but these are seldom classified as miracles. So, what exactly constitutes a miracle and does psi qualify?

To clarify the problem, consider the following possibility in alternate history. ${ }^{11}$ Recall that Einstein famously attacked the nonlocal implications of quantum mechanics, which he referred to as "spooky action at a distance." Consider Einstein using the same tactics as Reber and Alcock, insisting that the nonlocal behavior of quantum entanglement was impossible (violated relativity) and was therefore miraculous. (It seems plausible that many could have considered the violation of locality to be a violation of an established scientific principle in the same sense that Reber and Alcock try to establish in their paper.) Let's further suppose that Einstein simply dismissed any possible evidence supporting quantum entanglement. After all, he might 
argue, Hume advises us that the likelihood of fraud or poor methodology is greater than the violation of a miracle. So apparently, labeling something 'miraculous,' according to Reber and Alcock's interpretation of Hume, gives one license to dismiss the data, no matter how much has accumulated and no matter how many different tests are devised. Clearly, we need to take a closer look at what Hume meant by 'miracle.'

For Reber and Alcock, and other psi critics, David Hume's argument against miracles is a dependable weapon in the war against psi. ${ }^{12}$ I believe Hume's argument is indeed very formidable; however, I also believe the psi critics have used it carelessly. Hume's argument is aimed primarily at religious miracles, described in religious texts. These include such marvelous events as the dead rising from the grave, severed limbs growing back, the blind being cured of their blindness. The few non-religious miracles Hume mentions include lead floating in the air and sea monsters.

The heart of Hume's argument is the fallibility of human testimony. Hume lists the attributes of testimony that can be taken as credible and authoritative. These include testimony that agrees uniformly with other similar accounts, the manner of the delivered testimony, and the character providing the testimony. He puts great focus on the types of testimony that are uniform across many reports and that also agree with our own experiences. Hume then lists the ways that that testimony found in religious texts are found wanting. These typically arise in relatively remote areas where there are few witnesses. Testimony of a religious marvel often inspires a love of wonder or a kind of excited passion, and these he judges detrimental to more sober reasoning. Of course, he notes that dishonesty in testimony has occurred throughout history as well. Then Hume notes that religious miracles, such as raising the dead, clash strongly with the much more reasonable testimonies that we possess in much higher numbers and don't clash with our own experiences. Thus, the fallibility of human testimony leads one side to be much more lacking in evidence than the other.

But Hume wasn't addressing careful experimentation derived under laboratory conditions and ultimately evaluated through statistical techniques. This is something unacknowledged by the psi critics who borrow from Hume's argument. It is by no means clear how or why Hume's argument transfers to the laboratory, which employs methods to avoid the problems Hume describes, as well as sources of bias he doesn't.

However, while his argument is applied to the fallibility of testimony, Hume does provide us with something that does indeed have relevance for modern statistical evaluation: 
A wise man, therefore, proportions his belief to the evidence. In such conclusions as are founded on an infallible experience, he expects the event with the last degree of assurance, and regards his past experience as a full proof of the future existence of that event. In other cases, he proceeds with more caution: He weighs the opposite experiments: He considers which side is supported by the greater number of experiments: To that side he inclines, with doubt and hesitation; and when at last he fixes his judgement, the evidence exceeds not what we properly call probability. All probability, then, supposes an opposition of experiments and observations, where the one side is found to overbalance the other, and to produce a degree of evidence, proportioned to the superiority. A hundred instances or experiments on one side, and fifty on another, afford a doubtful expectation of any event; though a hundred uniform experiments, with only one that is contradictory, reasonably beget a pretty strong degree of assurance. In all cases, we must balance the opposite experiments, where they are opposite, and deduct the smaller number from the greater, in order to know the exact force of the superior evidence. (Hume, 1902, p. 50)

How is this inconsistent with the methods used to investigate psi? Cardeña summarizes more than 1,000 studies, which cover various modes of psi such as remote viewing and precognition. In some cases, the data are broken down into subsets to improve homogeneity. Combining the data of individual studies improves our ability to evaluate the probabilities that an effect might be real or spurious. The psi data, evaluated this way, escape the problems of fallible testimony that Hume describes.

But critics of psi would argue that Hume's argument implies that fraud is a greater likely explanation than some real effect. That is, psi skeptics tend to think that Hume's argument, which focused on the fallibility of testimony of a religious nature, can simply be transferred to the modern lab to account for findings that surprise them by invoking fraud. ${ }^{13}$ But such a step requires greater justification. How can we simply extend the notion of false witness regarding religious miracles that occurred hundreds or thousands of years ago toward modern scientists and statisticians exploring in an unconventional direction? Of course, fraud in parapsychology has occurred, although no more than in other fields. But it's important to note that such fraud was uncovered by other parapsychologists, peers of psi researchers reporting suspicious results. The results that Cardeña reports, involving more than 1,000 studies accumulated over decades in different laboratories, would require an unprecedented vast conspiracy among researchers. Scientists generally are wary of conspiracy theories for good reason. Obviously, it would be impossible to maintain such a conspiracy on this scale, with peers of psi researchers peeking over their shoulders.

But perhaps Reber and Alcock simply hold that psi phenomenon 
must be miraculous by definition. On the first page of their critique, they casually lump various modes of psi, such as precognition and ganzfeld, with non-laboratory examples of paranormal phenomenon, such as ghosts and tarot card readings. The authors make no effort to avoid conflating results obtained under controlled laboratory conditions, cumulated over decades, with other types of ostensible paranormal phenomena not addressed in Cardeña's overview. Perhaps Reber and Alcock are deliberately trying to muddy the picture. Or perhaps they simply view all of these phenomenon as the same kind of thing. Perhaps in their view, the laboratory data suggesting modest degrees of precognition or remote viewing can simply be lumped together with the sort of religious miracles that Hume dismissed. I would argue that the data Cardeña summarizes should stand on their own, without conflation with phenomena that are absent from the meta-analyses.

If we cast the notion of religious miracles aside, as well as other extraneous phenomenon not covered in the meta-analyses, what are we left with? Apparently, we have modes of anomalous cognition and perturbation with small effect sizes that are roughly in the ballpark with other psychological functions. Is there justification for placing these outside the boundary of what's possible? Or do we instead recognize these data, accumulated over decades under careful conditions, as simply something our current theories can't yet account for? I've already noted how the paradoxical behavior of quantum mechanics suggests interpretations that appear quite hospitable to psi. In the next section, I'll take up the problem of consciousness.

\section{The Persistent Mystery of Consciousness}

I've noted earlier that there is no known mechanism for how collections of non-conscious particles become conscious. This suggests consciousness would present a problem for at least one of Reber and Alcock's principles. In fact, the philosopher David Chalmers uses the notion of mechanism to help characterize what he terms the "hard problem of consciousness." According to Chalmers, the easy problems of consciousness are those that can be explained in terms of computational or neuronal mechanisms. The hard problem, on the other hand, is the problem of experience itself. Most philosophers of mind use a phrase suggested by Nagel (1974) to characterize this purely subjective aspect: there is something it is like to be a conscious organism.

This difficulty of putting subjective consciousness into some kind of analytical framework presents another problem for psi critics. Reber and Alcock consider it problematic that psi effects cannot be properly defined (p. 7). This is a critique that has come up before. As Alcock put it: “. . . 
unlike the various domains of mainstream science, it deals exclusively with phenomena that are only negatively defined" (Alcock, 2010, p. 33). But consciousness doesn't fare any better on this score if Nagel's characterization is the closest we can come to a definition. The phrase "there is something it is like to be a conscious organism" obviously doesn't identify anything we can objectively test. And that is indeed the chief problem of consciousness: Its inherently subjective nature makes it very difficult (perhaps impossible) to fit it into our objective understanding of the world.

Chalmers, Nagel, and others go so far as to argue that a purely physicalist or materialist framework cannot account for consciousness. They have deployed a number of philosophical arguments to make their case, which we don't have space for here. We can note, however, that there is nothing in all of the mathematical equations that comprise our understanding of physics that even remotely hint toward how consciousness arises from non-conscious particles. Philosophers of mind such as Chalmers and Nagel argue that consciousness most likely is fundamental in some sense, not emergent from matter. They see this move as necessary because they see consciousness as essentially anomalous with respect to our conventional, physicalist understanding. But such philosophers of mind typically have little interest in religion (or psi for that matter). They are simply putting alternatives on the table that might be better able to account for consciousness. The upshot apparently is that we must accept our subjective experiences as real, but recognize the difficulty accounting for them in a purely physical framework.

The grounds that Reber and Alcock use to characterize psi as a miraclebased on its difficulty of being integrated into our scientific worldviewlikely applies to consciousness. But if we accept our phenomenal experiences as real, the better choice would be to view consciousness as anomalous with respect to our conventional understanding. If we accept consciousness as anomalous, we are obviously on weak ground to dismiss other sorts of anomalous data closely linked to consciousness. And I see no reason to classify psi phenomenon as any more miraculous than our conscious experience. I submit that Reber and Alcock's characterization of psi as miraculous is unfounded. And a key component of their argument for dismissing the psi data collapses.

On the other hand, accepting the psi data may help us move forward in areas that we continue to struggle to understand. We might place less weight on interpretations of quantum mechanics that are inconsistent with psi. And our understanding of consciousness could be immeasurably deepened as well. While the psi data remain anomalous, the history of science suggests that this is simply not a good reason to ignore it. 


\section{Conclusion}

Reber and Alcock (2019a,b) take what must be considered an extreme position: Dismiss the psi data presented by Cardeña (2018) and others on the grounds that they run afoul of key scientific facts. However, their extreme position can't be justified, primarily because current theories in such areas as quantum mechanics and consciousness do not appear to be constrained by such facts. The fields of quantum mechanics and consciousness are not well-characterized through mechanistic approaches, which have been used so successfully in classical mechanics. And arguably, the psi data capture phenomenon relevant to both consciousness and quantum mechanics.

Also, I submit that psi skeptics should consider more carefully the problem of consciousness. Our consciousness appears to be anomalous with respect to our conventional understanding of the world. It also appears to be the kind of thing that would also fare poorly with a number of key criticisms aimed at psi. If we take our subjective experiences as anomalous, as many influential philosophers of mind suggest, are we truly in a position to dismiss the psi data?

\section{Notes}

1 The wave function consists of a large vector of possible observations for something of interest, such as the spin or position of a particle. And these possible observations are entangled with other possible observations of the quantum system.

2 In a later section, they criticize combining individual studies into metaanalysis on the grounds that such meta-analysis, based on flawed individual studies, must remain flawed. However, they fail to identify what flaws the original individual studies have.

3 Some have argued that the warm and noisy nature of our macro world likely rules out quantum effects. However, recent research in quantum biology challenges this view.

4 This may be an unfair comparison to the priests, who were not themselves claiming to be scientists.

5 There are various versions of Everett's interpretation, which I won't go into here. However, I believe I am capturing in broad strokes the rather straightforward version advocated by Carroll.

6 This has led Baggott (2019) in a recent essay to note that Carroll and other advocates of the multiverse practice "post-empirical science," which Baggott suggests can hardly be distinguished from pseudoscience. See Woit (2018) for another critical look at Carroll at https://www.math.columbia.edu/ woit/wordpress/?p=9938 
7 So while Carroll notes that life is too short to take every claim seriously, it is presumably not so short that he can't explore extravagant theories that we have no way of testing.

8 Skeptical bias and other limitations of the report have been documented by Palmer, Honorton, and Utts (1989), Bem and Honorton (1994), and Carter (2012).

9 The combined 90 studies of the meta-analysis in Bem et al. (2015) reported a $Z$ value of 6.40 and a $p$ value of $1.2 \times 10^{-10}$. By combining only those experiments associated with "thinking fast," the results were a $Z$ value of 7.11 and a $p$ value of $5.8 \times 10^{-13}$.

10 It is of course possible that individual studies could show little or only marginal statistical significance, yet would still contribute toward a very significant effect overall when combined into a meta-analysis, due to the relatively large statistical power that psi studies require. This is also the case with conventional psychology studies where effect sizes are relatively small or are influenced by a large number of factors.

11 I am indebted to Julia Mossbridge for suggesting this comparison.

12 Price (1955) was one of the first psi-skeptics to attack psi using Hume's argument. Hume's argument has also found its way into Wagenmakers et al.'s (2011) attack on Bem's (2011) findings of precognition.

13 I do not find in Reber and Alcock (2019a, 2019b) this argument explicitly; however. arguments of this sort can be found in Price (1955) and Wagenmakers et al. (2011).

\section{References}

Alcock, J. (2010). Attributions about impossible things. In. S. Krippner \& H. Friedman (Eds.), Debating psychic experience: Human potential or human illusion (pp. 29-42). Santa Barbara, CA: Praeger.

Baggott, J. (2019, October 7). But is it science? Aeon. Retrieved from https://aeon.co/essays/post-empirical-science-is-an-oxymoron-and-it-is-dangerous.

Bem, D. J. (2011). Feeling the future: Experimental evidence for anomalous retroactive influences on cognition and affect. Journal of Personality and Social Psychology, 100(3), 407.

Bem, D. J., \& Honorton, C. (1994). Does psi exist? Replicable evidence for an anomalous process of information transfer. Psychological Bulletin, 115(1), 4.

Bem, D. J., Tressoldi, P. E., Rabeyron, T., \& Duggan, M. (2015). Feeling the future: A meta-analysis of 90 experiments on the anomalous anticipation of random future events. F1000 Research, 4, 1188.

Bohm, D. (1986). A new theory of mind and matter. Journal of the American Society for Psychical Research 80, 113-135.

Bohm, D. (1996). Unfolding meaning: A weekend of dialogue with David Bohm. Abington, UK: Routledge.

Bohm, D., \& Hiley, B. J. (1993). The undivided universe: An ontological interpretation of quantum theory. London: Routledge.

Broderick, D., \& Goertzel, B. (Eds.). (2014). Evidence for psi: Thirteen empirical research reports. Jefferson, NC: McFarland. 
Cardeña, E. (2018). The experimental evidence for parapsychological phenomena: A review. American Psychologist,73(5), 663-677. doi:10.1037/amp0000236

Carroll, S. M. (2008, February 18). Telekinesis and quantum field theory (pp. 300-314). Retrieved from http://blogs.discovermagazine.com/cosmicvariance/2008/02/18/telekinesis-andquantum-field-theory/\#.XMcYnvZFxMs

Carroll, S. M. (2018). Beyond falsifiability: Normal science in a multiverse. In R. Dawid, R. Dardashti, \& K. Thébault (Eds.), Epistemology of fundamental physics: Why trust a theory? (p. 300). Cambridge UK: Cambridge University Press.

Carroll, S. M. (2019, September 7). Even physicists do not understand quantum mechanics. The New York Times. Retrieved from https://www.nytimes.com/2019/09/07/opinion/sunday/quantum-physics.html

Carter, C. (2012). Science and psychic phenomena: The fall of the house of skeptics. New York: Simon and Schuster.

Cramer, J. G. (1986). The transactional interpretation of quantum mechanics. Reviews of Modern Physics, 58(3), 647-687.

Druckman, D., \& Swets, J. (Eds.). (1988). Enhancing human performance: Issues, theories, and techniques. Washington, DC: National Academies Press.

Engber, D. (2017, May 17). Daryl Bem proved ESP is real: Which means science is broken. Retrieved from https://slate.com/health-and-science/2017/06/daryl-bem-proved-esp-is-realshowed-science-is-broken.html

Greenhouse, J. B. (1991). Comment: Parapsychology-On the margins of science. Statistical Science, 6(4), 386-389. http://dx.doi.org/10.1214/ss/1177011581

Hameroff, S., \& Penrose, R. (2014). Consciousness in the universe: A review of the 'Orch OR' theory. Physics of Life Reviews, 11(1), 39-78.

Hume, D. (1902). An enquiry concerning human understanding. Extracted from Enquiries concerning the human understanding, and concerning the principles of morals by David Hume. [Reprinted from the Posthumous Edition of 1777, L.A. Selby-Bigge (Ed.). Oxford, UK: Oxford University Press.] [Originally published 1748, retrieved from http://dx.doi.org/10.1093/oseo/instance.00032980

Kastner, R. E. (2012). The possibilist transactional interpretation and relativity. Foundations of Physics, 42(8), 1094-1113.

McFadden, J., \& Al-Khalili, J. (2016). Life on the edge: The coming of age of quantum biology. New York: Broadway Books.

Nagel, T. (1974). What is it like to be a bat? The Philosophical Review, 83(4), 435-450.

Palmer, J. A., Honorton, C., \& Utts, J. (1989, January). Reply to the National Research Council Study on parapsychology. Journal of the American Society for Psychical Research, 83(1), 31-49.

Price, G. R. (1955). Science and the supernatural. Science, 122(3165), 359-367.

Reber, A. S., \& Alcock, J. E. (2019a). Searching for the impossible: Parapsychology's elusive quest. American Psychologist, June 13. doi:10.1037/amp0000486

Reber, A. S., \& Alcock, J. E. (2019b). Why parapsychological claims cannot be true. Skeptical Inquirer, 43(4), 8-10.

Sheehan, D. P. (2015). Remembrance of things future: A case for retro-causation and precognition. In E. C. May \& S. B. Marwaha (Eds.), Extrasensory perception: Support, skepticism, and science (pp. 85-109). Santa Barbara, CA: Praeger.

Sokol, J. (2016, January 13). Time might flow forwards as well as backwards from the big bang. Retrieved from https://www.newscientist.com/article/mg22930564-100-time-mightflow-backwards-as-well-as-forwards-from-the-big-bang/

Stapp, H. P. (2017). Quantum theory and free will. Berlin: Springer.

Wagenmakers, E.-J., Wetzels, R., Borsboom, D., \& Van Der Maas, L. J. (2011). Why psychologists must change the way they analyze their data: The case of psi: Comment on Bem (2011). Journal of Personality \& Social Psychology, 100(3), 426-432.

Woit, P. (2018, January 17). Beyone falsifiability [blog post]. Not even wrong [blog]. Retrieved from https://www.math.columbia.edu/ woit/wordpress/?p=9938 